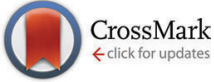

Cite this: J. Mater. Chem. B, 2016, 4, 2163

Received 7th January 2016 Accepted 24th February 2016

DOI: $10.1039 / c 6 t b 00052 e$

www.rsc.org/MaterialsB

\section{A switchable peroxidase mimic derived from the reversible co-assembly of cytochrome $c$ and carbon dots $\dagger$}

\author{
Jeremy B. Essner, Richard N. McCay, Chip J. Smith II, Stephen M. Cobb, \\ Charles H. Laber and Gary A. Baker*
}

\begin{abstract}
We describe a straightforward tactic to boost the inherently low peroxidase-like activity of the hemeprotein equine cytochrome $c$ (cyt c) following its electrostatic assembly onto the carbon nanodot surface. This represents the first time that carbon nanodot interaction has been demonstrated to switch a protein into a high-performance enzyme for speeding up a reaction it was not evolved to catalyze. The dramatic enhancement in peroxidase-like activity stems in part from favorable local perturbations within the heme microenvironment of cyt $c$ which are influenced by the chemistry presented at the carbon dot surface. That is, the observed peroxidase activity is clearly moderated by the choice of molecular precursors used to prepare the carbon dots, a choice which ultimately determines the surface charges present. An exceptional catalytic efficiency $\left(k_{\text {cat }} / K_{M}\right)$ of $8.04( \pm 1.74) \times 10^{7} \mathrm{M}^{-1} \mathrm{~s}^{-1}$ was determined for carbon dot/cyt $c$ co-assemblies, close to the theoretical diffusion-controlled limit. Notably, the activity of the carbon dot/cyt $c$ assembly can be switched off simply by increasing the ionic strength which results in dissociation into non-catalytic components.
\end{abstract}

\section{Introduction}

With the development of novel nanomaterials that show promise in biological applications, it is vital to arrive at a deeper understanding of these nanomaterials' interactions and effects on the biological systems in which they may be applied. Toward this, it has been reported that various nano-sized materials possess the ability to enhance or hinder enzymatic activities across various research realms such as catalysis and the detection of biological targets. ${ }^{1-3}$ Even further and as a greener alternative to conventional nanomaterials, carbon-based nanomaterials such as graphenes and fullerenes are gaining traction as protein/ biomolecule sensors, enzyme modulators, and intracellular transporters due to their biocompatibility and desirable surface functionalities. $^{2,4-9}$

Recently, Yang et al. demonstrated that the peroxidase-like activity of cytochrome $c$ (cyt $c$ ) was modulated through the addition of reduced graphene oxide (rGO) or graphene oxide (GO), which resulted in inhibited or enhanced activity, respectively. ${ }^{2}$ The observed activity enhancement resulting from the addition of GO was attributed to the perturbation of the cyt $c$ heme

Department of Chemistry, University of Missouri-Columbia, Columbia, MO 65211, USA. E-mail: bakergar@missouri.edu

$\dagger$ Electronic supplementary information (ESI) available: Full experimental details, characterizations, and supporting figures. See DOI: 10.1039/c6tb00052e microenvironment through electrostatic interactions between the protein and GO functional groups. These functional groups (e.g., epoxide, carboxyl, hydroxyl) have been reported to covalently bond, hydrogen bond, or electrostatically interact with proteins, resulting in efficient biosensors or enzymatic modulators. ${ }^{2,4,5}$

Carbon nanodots (C-dots), emerging carbon nanomaterials, are thought to possess similar surface functionalities to $\mathrm{GO},{ }^{10}$ making them intriguing nanoparticle candidates for such enzymatic modulation. In addition to these surface features which impart C-dots with high aqueous solubility, C-dots also sport several other attractive and exploitable properties (especially in relation to bio-applications), such as biocompatibility, low cytotoxicity, excitation wavelength-dependent emission, and relatively high luminescence quantum yields. ${ }^{10,11}$ One other appealing feature of C-dots is the ability to generate this nanomaterial from virtually any carbon-containing source, including low- and negatively-valued wastes (even human urine ${ }^{12}$ ) through an arsenal of top-down (e.g., arc discharge, laser ablation, electrochemical oxidation) and bottom-up (e.g., thermal, hydrothermal, and microwave) approaches. ${ }^{10}$

While C-dots appear to be interesting candidates for efficient enzymatic modulation, past protein-related C-dot work has focused primarily on protein detection ${ }^{13-15}$ or insulin fibrillation inhibition, ${ }^{16}$ with few reports relevant to C-dot modulated enzyme activity, the nature of protein/C-dot interactions, or the effects of C-dots on protein structure. ${ }^{3,17,18}$ For example, Li et al. 
demonstrated that phosphate-functionalized C-dots enhance the catalytic activity of laccase through interactions between the C-dot surface groups and the protein's $\mathrm{T} 1 \mathrm{Cu}$ center, with photoirradiation of the system further enhancing the activity. ${ }^{3}$ Herein, we report, for the first time, the C-dot modulated peroxidase-like activity of cyt $c$. Cyt $c$, a globular protein with a unique covalently-bound heme center, serves as an excellent model protein to assess local perturbations arising from protein/ C-dot interactions, since the effects on the active heme center can be probed spectroscopically and visualized through peroxidasemimicking catalysis. ${ }^{1,2}$ Although cyt $c$ is a known peroxidase mimic, the inherently-low activity limits its widespread implementation in catalytic applications, making the development of efficient nanoscale 'boosters' of high interest.

\section{Results and discussion}

In this work, four different types of C-dots were synthesized and tested for their ability to enhance the peroxidase-like activity of cyt $c$. The detailed synthetic procedures are provided within the Experimental but briefly, citric acid, as a carbon source was thermally carbonized alone and in the presence of various dopants/passivating agents, ${ }^{19,20}$ resulting in citric acid (CA), citric acid-urea (CA-U), citric acid-thiourea (CA-T), and citric acidmercaptosuccinic acid (CA-M) derived C-dots. ${ }^{21}$ To the best of our knowledge, this represents the first example wherein C-dots have been shown to repurpose a protein to perform a catalytic reaction it was not evolved to perform (i.e., non-native biocatalysis).

The cartoon schematic ${ }^{22}$ shown in Fig. 1 highlights the postulated interactions between the CA-derived C-dots and cyt $c$. In the presence of only CA C-dots, hydrogen peroxide $\left(\mathrm{H}_{2} \mathrm{O}_{2}\right)$ was unable to oxidize the leuko-dye 2,2'-azino-bis(3-ethylbenzothiazoline-6-sulphonic acid) (ABTS), indicated by the absence of green color (Fig. 1Ai). Consistent with previous reports, cyt $c$ alone showed minimal peroxidase activity, slowly oxidizing ABTS (Fig. 1Aii). ${ }^{1,2}$ However, in the presence of C-dots, the peroxidase-like activity of cyt $c$ was markedly enhanced, denoted by the rapid formation of the characteristic green color for oxidized ABTS (Fig. 1Aiii). As we will demonstrate in this study, this activity modulation can best be explained through an electrostatically-driven perturbation of the cyt $c$ heme center by the C-dots. The surface functionality of the C-dots consists of carboxyl, hydroxyl, and epoxide moieties, as determined by infrared spectroscopy (Fig. S1B, ESI $\dagger$ ), which yields a negativelycharged surface. Meanwhile, cyt $c$ possesses a largely cationic surface ( $\mathrm{pI} \approx 10.3$ ), with much of that positive charge residing proximal to the active heme site, as shown by the electrostatic calculations of Fig. 1B. ${ }^{22-24}$ Therefore, we postulate that the cyt $c$ "docks" with the C-dot surface preferentially near its heme site, resulting in a perturbation of the heme microenvironment of cyt $c$ giving rise to peroxidase-like activity.

\section{Carbon nanodot characterization}

The photophysical and physical properties of the various C-dots were characterized with UV-Vis, Fourier-transform infrared,

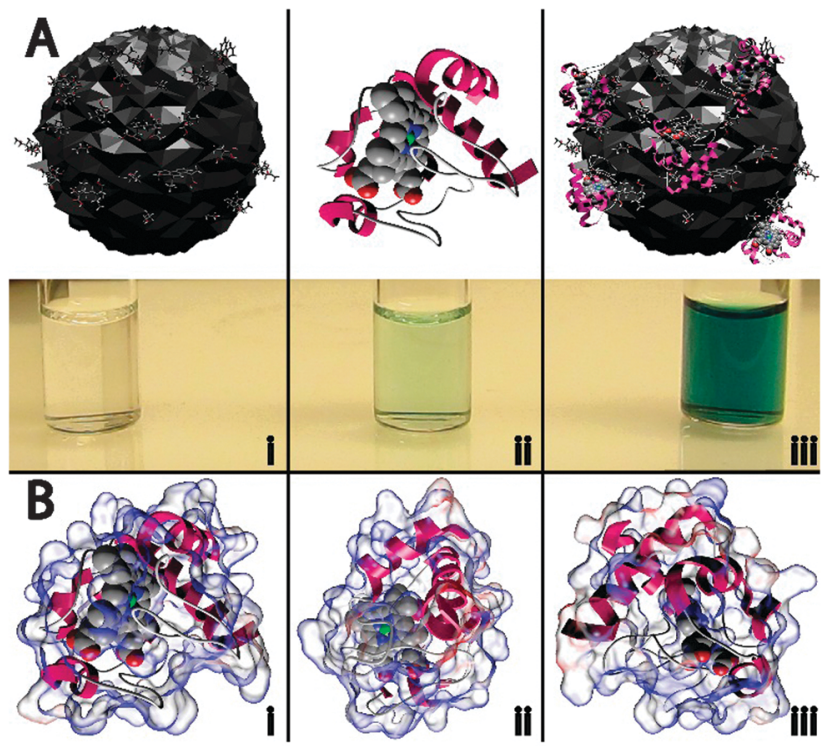

Fig. 1 (A) Neat carbon nanodots (C-dots) did not display any catalytic activity, as evidenced by the lack of green color in (Ai), while native cytochrome $c$ (cyt $c$ ) was found to exhibit weak peroxidase activity (Aii). When $C$-dots were combined with cyt $c$, the peroxidase activity was dramatically enhanced, indicated by the rapid formation of the oxidized form of ABTS (i.e., appearance of intense green color), as shown in (Aiii). (B) Different orientations of the electrostatic calculations performed on cyt $C$ show that a majority of the protein surface is positively charged (blue areas), with the bulk of the positive charge residing in the vicinity of the heme group.

and fluorescence spectroscopies, as well as transmission electron microscopy. The UV-Vis spectra of the four C-dot samples are provided in Fig. S1A (ESI $\dagger$ ). The CA and CA-M derived C-dots display rapidly increasing absorbance in the near-UV region, which is characteristic of the $\pi-\pi^{*}$ transitions of aromatic $\mathrm{sp}^{2}$ domains. ${ }^{21}$ On the contrary, the CA-U and CA-T derived C-dots show small peaks at approximately $340 \mathrm{~nm}$, likely due to nitrogen incorporation within the C-dot. In addition, the CA-T derived C-dots display an additional peak centered near $450 \mathrm{~nm}$.

The FTIR spectra (Fig. S1B, ESI $\dagger$ ) reveal that, depending upon the precursors employed, the C-dots possess hydroxyl, carboxyl, epoxide, and amine functionalities, which were assigned to the following absorption bands: $3400 \mathrm{~cm}^{-1}$, stretching vibrations of $\mathrm{O}-\mathrm{H}\left(\nu_{\mathrm{O}-\mathrm{H}}\right)$ and/or $\mathrm{N}-\mathrm{H}\left(\nu_{\mathrm{N}-\mathrm{H}}\right) ; 1700$ and $1650 \mathrm{~cm}^{-1}$, stretching vibrations of $\mathrm{C}=\mathrm{O}\left(\nu_{\mathrm{C}}=\mathrm{O}\right) ; 1650-1550 \mathrm{~cm}^{-1}, \mathrm{NH} / \mathrm{NH}_{2}$ deformation $\left(\nu_{\mathrm{N}-\mathrm{H}}\right)$ and skeletal vibrations of aromatic groups $\left(\nu_{\mathrm{C}}=\mathrm{C}\right) ; 1300-1000 \mathrm{~cm}^{-1}$, stretching vibrations of $\mathrm{C}-\mathrm{O}-\mathrm{C}$ and $\mathrm{C}-\mathrm{OH}\left(\nu_{\mathrm{C}-\mathrm{O}-\mathrm{C}}\right.$ and $\left.\nu_{\mathrm{C}-\mathrm{OH}}\right) ; 2900$ and $1400 \mathrm{~cm}^{-1}$, aliphatic stretches $\left(\nu_{\mathrm{C}-\mathrm{H}}\right)$. Due to the presence of these surface functionalities, all of the resulting dots were highly water-soluble. Fluorescent emission spectra (Fig. S2, ESI $\dagger$ ) of the CA, CA-U, CA-T, and CA-M derived C-dots show that, in general, upon increasing the excitation wavelength, the fluorescent emission maxima display a slight hypsochromic shift, followed by a larger bathochromic shift. These substantial red-edge effects are further highlighted in the inset plots of Fig. S2 (ESI $\dagger$ ). Excitation wavelength-dependent quantum yield measurements (Fig. S1C, ESI $\dagger$ ) reveal that the 
quantum yield values decreased as the excitation wavelength increased, with the CA and CA-T derived C-dots displaying the highest overall quantum yields across all wavelengths. Representative TEM images (Fig. S1D, ESI $\dagger$ ) of the CA-derived $\mathrm{C}$-dots show that the dots ranged in size from $30-60 \mathrm{~nm}$, with particles as large as $100 \mathrm{~nm}$ seen on rare occasion. All other precursor combinations produced C-dots of a similar size range.

\section{Carbon nanodot screening}

Fig. 2A reveals that while native cyt $c$ displays very weak peroxidase-like activity, by themselves, the C-dots display no intrinsic peroxidase activity. However, when C-dots are introduced to cyt $c$, the peroxidase-mimicking activity of the protein is greatly enhanced. Moreover, this effect is highly dependent on the nature of the C-dot chemistry. Since the largest modulation arises from CA and CA-M, we postulate that more negativelycharged C-dots produce greater peroxidase activity enhancements due to electrostatically-driven coupling with cyt $c$. Indeed, C-dots derived from $\mathrm{CA}-\mathrm{U}$ and $\mathrm{CA}-\mathrm{T}$ are expected to possess cationic
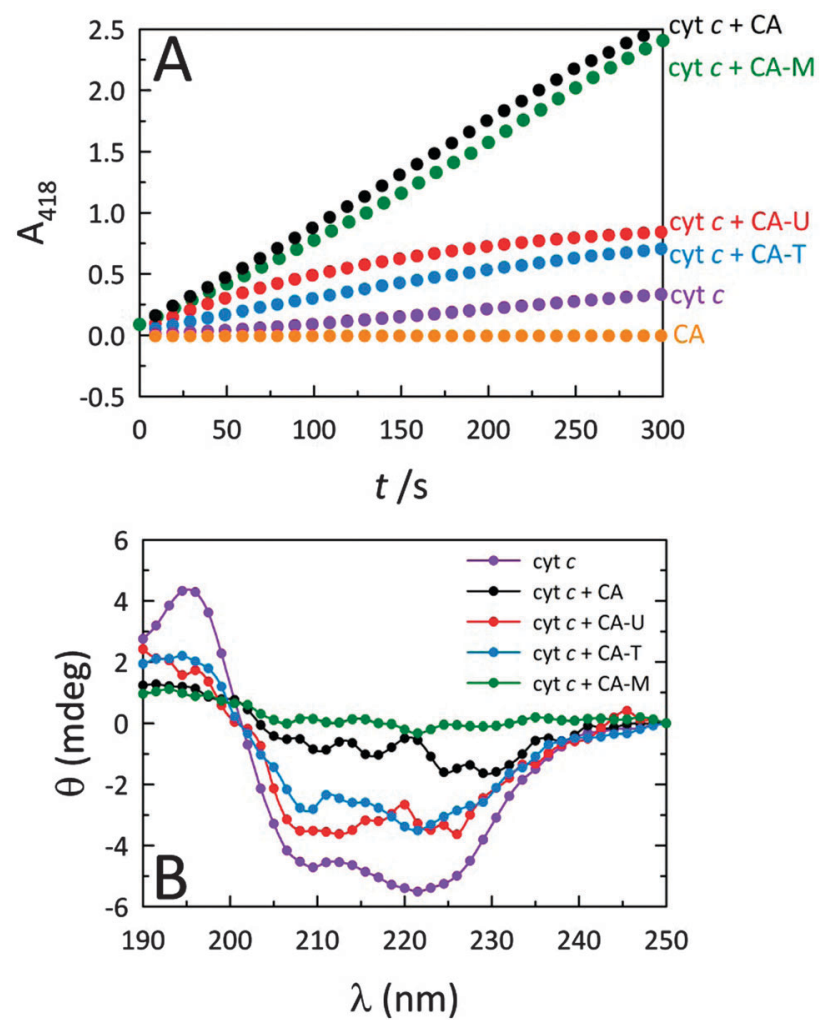

Fig. 2 (A) Peroxidase-like activities of native cyt $c$ and cyt $c$ in the presence of various $\mathrm{C}$-dots. The chemistry of the $\mathrm{C}$-dot has a marked impact on the observed peroxidase-like cyt $C$ activity, with $C A$ and $C A-M$ derived $\mathrm{C}$-dots yielding the greatest catalytic enhancements. Typical assay conditions: [cyt $\mathrm{c}$ ] $=515 \mathrm{nM},\left[\mathrm{C}\right.$-dot] $=25.5 \mu \mathrm{g} \mathrm{mL}^{-1},\left[\mathrm{H}_{2} \mathrm{O}_{2}\right]=14.1 \mathrm{mM}$, [ABTS] $=0.72 \mathrm{mM}$. (B) Far-UV CD spectra of cyt $c$ alone and in the presence of the four different types of $\mathrm{C}$-dots. These spectra illustrate the role that the $\mathrm{C}$-dot surface functionalities play in denaturing cyt $c$. The degree to which the protein is denatured correlates with the peroxidase activity shown in panel $(A)$, with $C A$ and $C A-M$ dots showing the greatest structural impact coupled with the highest peroxidase activity. Representative $C D$ conditions were: [cyt $c]=4.2 \mu \mathrm{M}$ and $\left[C\right.$-dot] $=200 \mu \mathrm{g} \mathrm{mL}^{-1}$. surface moieties arising from the (thio)urea component, consistent with the significantly lower peroxidase activities observed for those C-dots. The circular dichroism (CD) measurements summarized in Fig. 2B correlate well with the corresponding activities shown in Fig. 2A, consistent with the notion that the degree of protein perturbation, which depends on the features of the dots (e.g., surface charge), determines the peroxidase activity.

\section{CA carbon nanodot concentration optimization}

To better understand the observed peroxidase activity modulation for cyt $c$, the interactions between CA-derived C-dots and cyt $c$ were explored more fully since these particular C-dots delivered the highest activity. For the remainder of this article, 'C-dots' will refer solely to CA-derived C-dots, unless otherwise noted. Initially, the C-dot loading was optimized by monitoring the peroxidase activity for cyt $c$ in the presence of various C-dot concentrations. Fig. 3 shows that a very low C-dot loading of $0.10 \mu \mathrm{g} \mathrm{mL}{ }^{-1}$ produced a small, but statistically-significant, enhancement in activity over native cyt $c$, with the activity increasing greatly at higher C-dot loadings. A sharp increase in the peroxidase-like activity of cyt $c$ was observed upon increasing the C-dot concentration from 5.0 to $10.0 \mu \mathrm{g} \mathrm{mL}{ }^{-1}$, however, C-dot loadings higher than $10.0 \mu \mathrm{g} \mathrm{mL}^{-1}$ did not provide any further enhancements. For the remaining studies, we have employed a C-dot concentration of $25.0 \mu \mathrm{g} \mathrm{mL}$ as this gave near-peak activities wherein the cyt $c$ active site appeared to be 'saturated' with respect to C-dot interactions.

\section{Iron ion and molecular control studies}

Also summarized in Fig. 3 are the results of control studies in which free iron (in the form of either $\mathrm{Fe}^{2+}$ or $\mathrm{Fe}^{3+}$ ) was tested for peroxidase activity. In both cases, negligible peroxidase activity was shown compared to native cyt $c$, ruling out the possibility that expulsion of iron from the heme group might contribute to the emergent peroxidase activity. These results further highlight the pivotal role that the C-dots play in turning on the peroxidase-like activity of cyt $c$.

In addition to these iron controls, various molecular analogs were tested for their ability to induce cyt $c$ peroxidase activity via electrostatic interactions. For these studies, the C-dot precursors themselves (i.e., citric acid, urea, thiourea, mercaptosuccinic acid) as well as tannic acid and $10 \mathrm{kDa}$ polyethylenimine (PEI) were tested as model species since they provide a range of molecular sizes as well as surface groups (i.e., charges). The concentration of the model species tested was a decade lower than the C-dot concentration (on a mass basis), to account for the fact that less than $10 \%$ of the C-dot atoms are actually surface exposed. Addition of these test molecules to cyt $c$ produced weak or zero enhancement in the peroxidase-like activity (Fig. S3, ESI $\dagger$ ). Citric acid produced the highest degree of enhancement. Urea, thiourea, and mercaptosuccinic acid produced similar enhancements, although the mercaptosuccinic acid resulted in an initial induction time prior to catalytic rate increase. The $10 \mathrm{kDa}$ PEI gave no enhancement, in line with an electrostatic interaction mechanism (i.e., mutual repulsion between the positively-charged protein and positivelycharged polymer). Surprisingly, however, tannic acid, which 

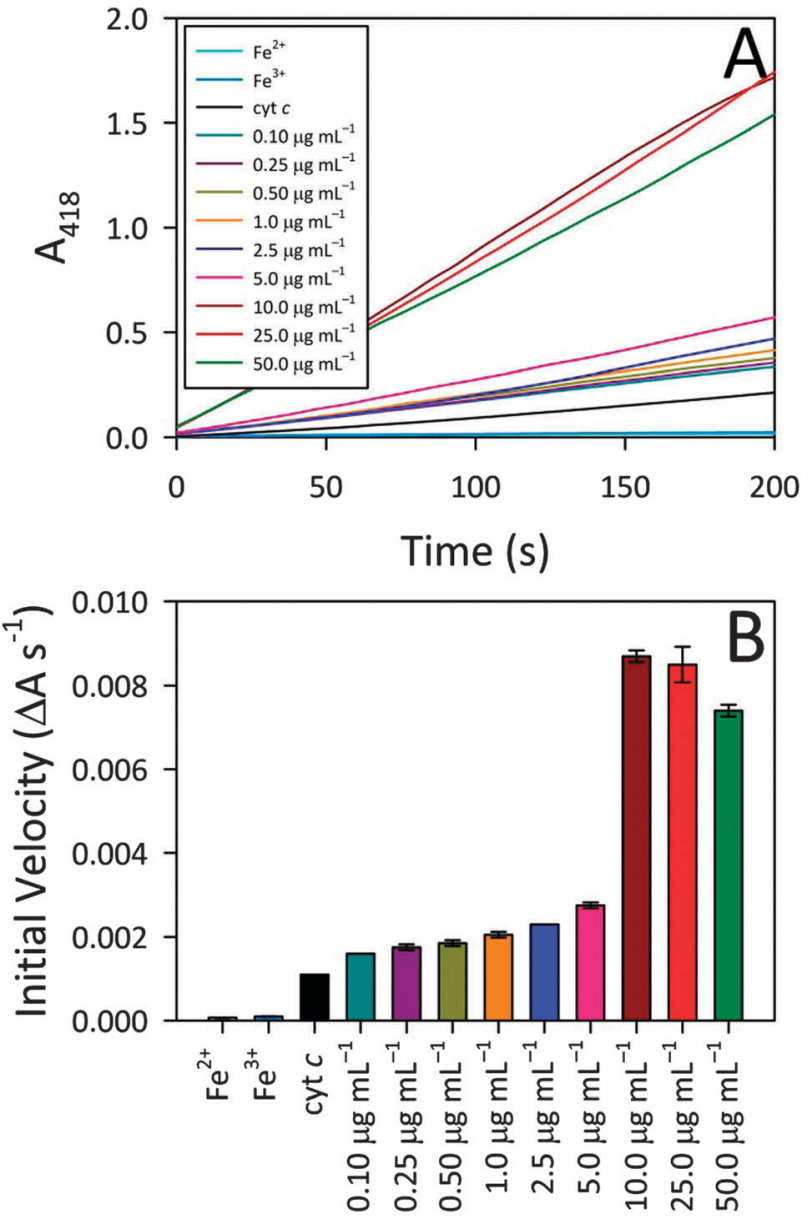

Fig. 3 (A) CA-derived C-dot concentration dependence of observed cyt C peroxidase activity, demonstrating that increasing $\mathrm{C}$-dot concentration leads to higher activity. Cyt $\mathrm{C}$ and free iron ( $\mathrm{Fe}^{2+}$ and $\mathrm{Fe}^{3+}$ ion) controls are included alongside for comparison. (B) Calculated slopes from the activity profiles of panel (A) further highlight that increasing the C-dot concentration yields higher cyt $c$ peroxidase activity whereas free iron itself shows essentially zero peroxidase activity. Typical assay conditions: [cyt $\mathrm{C}, \mathrm{Fe}^{2+}$, or $\left.\mathrm{Fe}^{3+}\right]=515 \mathrm{nM},[\mathrm{C}$-dot $]=$ variable (as shown), $[\mathrm{ABTS}]=0.72 \mathrm{mM}$, and $\left[\mathrm{H}_{2} \mathrm{O}_{2}\right]=14.1 \mathrm{mM}$.

possesses negatively-charged groups, also yielded no activity. In fact, the activity of cyt $c$ in the presence of tannic acid is lower than for cyt $c$ alone. Overall, these control studies point to the fact that, while electrostatic interactions appear to play an important role, they cannot fully account for the observed peroxidase activities. That is, the nanoscale dimensions of the C-dots also appear to play a vital part in the protein structure perturbation giving rise to exceptional peroxidase-mimicking activity (i.e., the physical docking of cyt $c$ onto C-dots).

\section{Peroxidase kinetics studies and ionic strength effects}

To further describe peroxidase performance, results were placed within a Michaelis-Menten kinetic framework. Toward this, C-dots were mixed with increasing concentrations of the ABTS substrate to obtain saturation plots of cyt $c$ peroxidase activity (Fig. 4A). The initial reaction velocities were plotted against the substrate (ABTS) concentration (Fig. S4A, ESI $\dagger$ ),
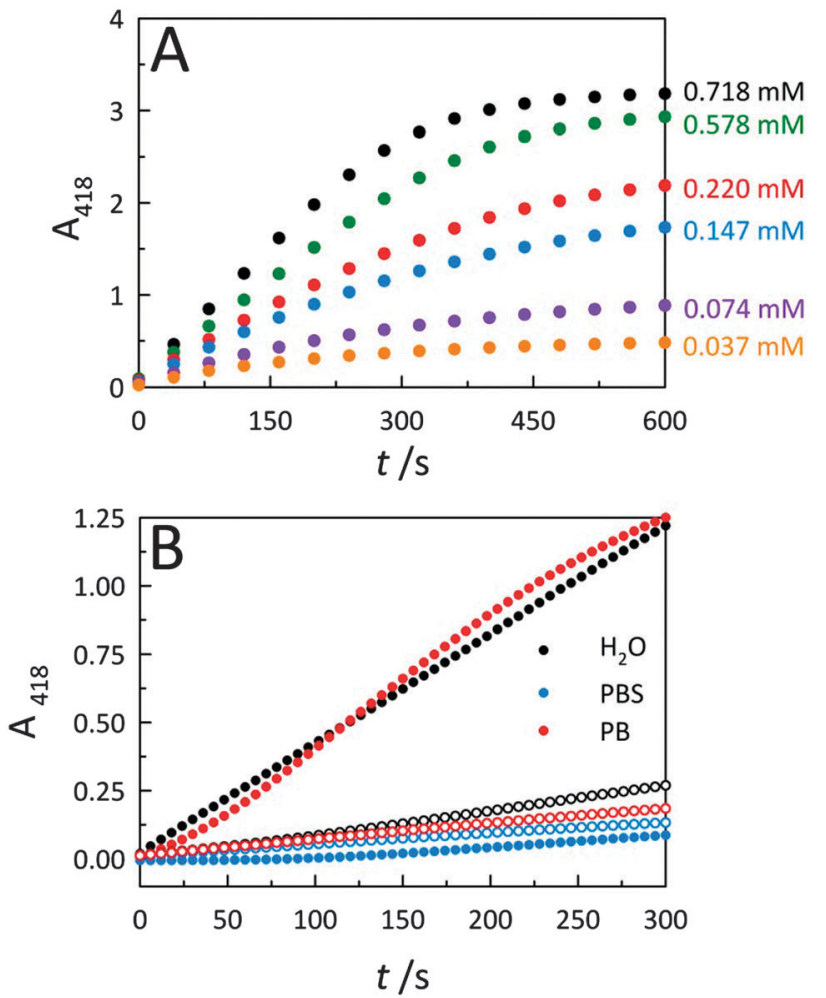

Fig. 4 (A) CA-derived C-dot modulated peroxidase activity for cyt $c$ in the presence of increasing ABTS substrate concentrations. (B) Solvent system effects on cyt $c$ peroxidase-like activities in the presence (solid circles) and absence (open circles) of CA-derived C-dots. Use of a phosphate buffered saline (PBS) solvent system resulted in a drastic reduction in peroxidase activity, even in the presence of $\mathrm{C}$-dots, consistent with the interaction between cyt $\mathrm{C}$ and $\mathrm{C}$-dots being electrostatic in nature. Typical assay conditions employed were $[$ cyt $\mathrm{c}]=515 \mathrm{nM},[\mathrm{C}$-dot $]=25.5 \mu \mathrm{g} \mathrm{mL}^{-1},\left[\mathrm{H}_{2} \mathrm{O}_{2}\right]=14.1 \mathrm{mM}$.

revealing biocatalytic behaviour that follows Michaelis-Menten kinetics, similar to observations for cyt $c$ on GO. ${ }^{2}$ Using these same data, a Lineweaver-Burk plot (Fig. S4B, ESI $\dagger$ ) was generated to determine the characteristic catalytic parameters associated with the observed enzyme kinetics, including the maximum velocity of the enzymatic reaction $\left(V_{\max }\right)$, the Michaelis constant $\left(K_{\mathrm{M}}\right)$, the turnover number $\left(k_{\text {cat }}\right)$, and the catalytic efficiency (defined as $k_{\text {cat }} / K_{\mathrm{M}}$ ), a measure of how efficiently an enzyme converts a substrate into product. From the LineweaverBurk plot, the $V_{\text {max }}, K_{\mathrm{M}}, k_{\text {cat }}$, and $k_{\text {cat }} / K_{\mathrm{M}}$ values were calculated as $0.010 \pm 0.002 \mathrm{~s}^{-1}, 0.245 \pm 0.021 \mathrm{mM}, 1.97( \pm 0.39) \times 10^{4} \mathrm{~s}^{-1}$, and $8.04( \pm 1.74) \times 10^{7} \mathrm{M}^{-1} \mathrm{~s}^{-1}$, respectively. This catalytic efficiency approaches the theoretical upper limit for diffusion of substrate into an active site $\left(10^{8}-10^{10} \mathrm{M}^{-1} \mathrm{~s}^{-1}\right)$, an indicator of the outstanding efficiency for peroxidase activity displayed by co-assemblies of cyt $c$ with C-dots.

To further elucidate the nature of the interaction between C-dots and cyt $c$, catalytic studies were carried out within three different aqueous solvent systems: water, phosphate buffer (PB), and phosphate buffered saline (PBS) (Fig. 4B). Whether performed in deionized water (i.e., ionic strength, $\mu \approx 0$ ) or $\mathrm{PB}$ (10 mM, pH 7.4; $\mu \approx 0.02 \mathrm{M}$ ), C-dots were found to similarly enhance the peroxidase activity of cyt $c$. Conversely, upon 
increasing the ionic strength of the solvent system by conducting the catalysis in PBS which contains supplemental salt (ionic strength, $\mu \approx 0.16 \mathrm{M}$ ), the activity falls off completely to levels below that of the cyt $c$ alone. This loss in peroxidase activity is fully consistent with the increased ionic strength resulting in a dissociation of the catalytically-active electrostatic assembly. Namely, the Coulombic screening removes the attraction between cyt $c$ and the oppositely-charged C-dot surface. Now detached from the C-dot surface, the cyt $c$ no longer displays strong peroxidase activity.

\section{Probing the heme microenvironment}

To further illuminate the origin of this enzymatic modulation, the absorbance of cyt $c$ at $695 \mathrm{~nm}$ was monitored upon the addition of C-dots. It is well established that cyt $c$ presents a characteristic absorption band centered at $695 \mathrm{~nm}$ which is a sensitive marker for the native protein conformation. ${ }^{25}$ The loss of this band is typically associated with a perturbation of the heme microenvironment (weakening or rupture of the axial Met80- $\mathrm{Fe}^{3+}$ bond), resulting in an opening of the heme crevice. ${ }^{25}$ The UV-Vis results shown in Fig. S5 (ESI $\dagger$ ) indicate that, upon addition of C-dots to a native solution of cyt $c$, the 695 band is completely lost, providing clear evidence that C-dots perturb the heme environment of cyt $c$. The fact that the positive surface charges of cyt $c$ are concentrated near the heme group likely directs the electrostatic association between cyt $c$ and C-dots to occur at this locus, yielding both global and local (heme) structural changes within cyt $c$ which form the genesis of the strong peroxidase-like activity shown by the co-assembly.

It has been reported that a perturbed heme environment, and/or losses in alpha $(\alpha)$ helical content within cyt $c$, can result in increased peroxidase-like activity. ${ }^{26-29}$ Circular dichroism (CD) spectroscopy provides a convenient means to monitor such protein conformational changes. The CD measurements presented in Fig. 2B and Fig. 5 support the notion that, in the presence of a sufficient concentration of C-dots, the secondary structure of cyt $c$ is perturbed to the point of losing most of its $\alpha$-helical character. ${ }^{30}$ Upon further analysis using secondary structure prediction methods, ${ }^{31}$ it was found that the approximate $\alpha$-helical content of the protein decreased from an initial value of $24 \%$ to just $2.3 \%$ upon the addition of CA-derived C-dots. Meanwhile, the random content rose from 34\% to $41 \%$. These results indicate that the global protein fold is seriously altered and, in concert with changes local to the heme group, is responsible for the large catalytic enhancement. Fig. 5B reveals substantial changes in the Soret region of the CD spectrum as well, indicating that the heme microenvironment is critically altered in the presence of C-dots. ${ }^{32}$ In particular, the Soretregion $\mathrm{CD}$ spectrum of cyt $c$ in the presence of $\mathrm{C}$-dots no longer retains the characteristic dichroic signature of ferri-cyt $c$.

\section{Conclusions}

We have demonstrated that C-dots can function as efficient enzyme modulators to induce strong peroxidase activity in cyt $c$.
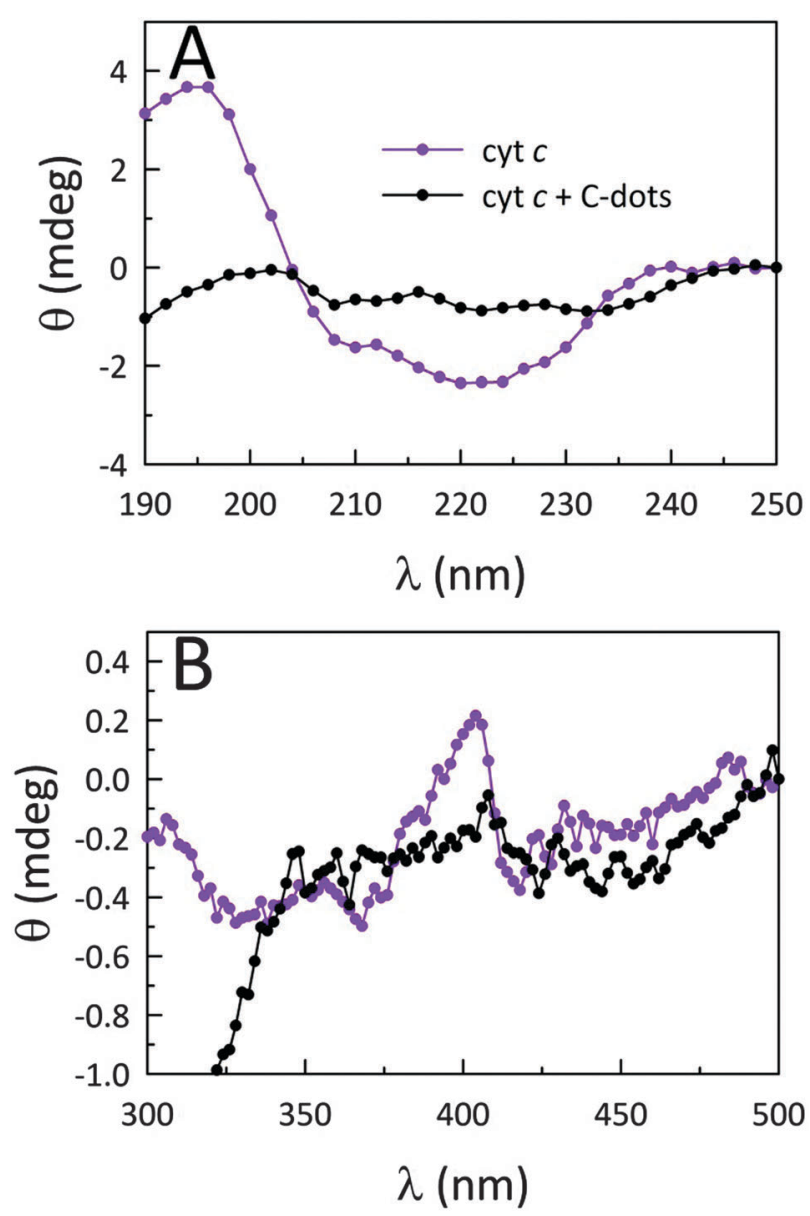

Fig. 5 (A) Far-UV CD spectra of cyt $C$ in the presence of CA-derived C-dots; [cyt c] $=4.2 \mu \mathrm{M}$ and [C-dot] $=200 \mu \mathrm{g} \mathrm{mL}{ }^{-1}$. It is evident that the secondary structure of cyt $c$ is completely altered by the introduction of C-dots. (B) The local heme environment of cyt $c$ is also disturbed by the presence of C-dots, as seen in the diminished Soret band at $410 \mathrm{~nm}$.

Remarkably, by electrostatic assembly of cyt $c$ onto oppositelycharged C-dots, the cyt $c$ becomes an efficient biocatalyst for a reaction it was not evolved to perform. The emergent peroxidase activity is concomitant with significant changes in the protein secondary structure as well as alterations within the local environment surrounding the heme prosthetic group. This modulated behaviour is dependent upon the surface chemistry (particularly, surface charge) of the C-dots, with anionic dots derived from citric acid or citric acid/mercaptosuccinic acid imparting the highest peroxidase-like activity to cyt $c$. Control experiments suggest that, while electrostatic interactions are key, they may not be the sole contributor giving rise to strong peroxidase mimics and that the physical co-assembly at a nanoscale (high curvature) surface also appears to play a role. This work adds to our growing understanding of the interactions between nanocarbons and enzymes and suggests enticing prospects for nanocarbonbased modulation of other potential Fe-centered peroxidase mimics (e.g., CaHydA), ${ }^{33}$ with implications in bionanochemistry, sensing, and biocatalysis. 


\section{Experimental}

\section{Materials and reagents}

All experiments were carried out using Ultrapure Millipore water $(18.2 \mathrm{M} \Omega \mathrm{cm})$. Equine heart cytochrome $c(\mathrm{C} 7752,>95 \%)$, hydrogen peroxide (216763, $30 \mathrm{wt} \%$ ), 2,2'-azino-bis(3-ethylbenzothiazoline-6-sulfonic acid) diammonium salt (ABTS, 11557, 98\%), citric acid monohydrate (C0706, $\geq 99.5 \%)$, urea (51459, $\geq 99.5 \%$ ), thiourea (T7875, $\geq 99.0 \%)$, mercaptosuccinic acid (88460, $\geq 99.0 \%$ ), phosphate buffered saline (PBS) tablets (P4417), sodium phosphate monobasic monohydrate $(71507, \geq 99.5 \%)$, sodium phosphate dibasic (71636, $\geq 99.5 \%$ ), sodium chloride (S7653, $\geq 99.5 \%$ ), potassium bromide (451010, 99.95\%), quinine hemisulfate (quinine sulfate) salt monohydrate $(22640, \geq 98.0 \%$ ), fluorescein sodium salt (F6377), and tannic acid (16201) were all purchased from Sigma-Aldrich (St. Louis, MO) and used as received. Rhodamine 610 (rhodamine B) chloride (06101) was acquired from Exciton, Inc (Dayton, OH). Polyethylenimine (PEI) (19850, 99\%; $10 \mathrm{kDa}$ molecular weight) was purchased from Polysciences, Inc. (Warrington, PA). The chloride salts of $\mathrm{Fe}^{3+}$ (AC423705000, hexahydrate, 97+\%) and $\mathrm{Fe}^{2+}$ (AC389350250, anhydrous, 99\%) were obtained from Fisher Scientific (Pittsburg, PA). Cellulose dialysis tubing (132105, Spectra/Por 7, $1.0 \mathrm{kDa}$ molecular weight cut-off, MWCO) was acquired from Spectrum Labs.

\section{Characterization techniques}

UV-Vis absorbance spectra and data for the peroxidase activities and ABTS kinetic measurements were acquired on a Hitachi U-3000 spectrophotometer. All other absorbance spectra and fluorescence data were collected using a Cary Bio 50 UV-Vis spectrophotometer and a Varian Cary Eclipse Fluorometer, respectively. Quantum yield values were calculated based on the expression listed below with quinine sulfate (QS), fluorescein (FL), and rhodamine $\mathrm{B}(\mathrm{RB})$ as reference fluorophores (fluorophore and fluorescence measurement information is provided below in Table 1).

$$
\mathrm{QY}_{\mathrm{S}}=\mathrm{QY}_{\mathrm{R}}\left(\frac{F_{\mathrm{S}}}{F_{\mathrm{R}}}\right)\left(\frac{\mathrm{OD}_{\mathrm{R}}}{\mathrm{OD}_{\mathrm{S}}}\right)\left(\frac{n_{\mathrm{S}}^{2}}{n_{\mathrm{R}}^{2}}\right)
$$

Here, $\mathrm{R}$ and $\mathrm{S}$ represent reference and sample, respectively, $F$ is the integrated fluorescence intensity (calculated over the wavelength range of interest), OD stands for optical density (at the excitation wavelength used in the fluorescent measurements), and $n$ is the refractive index of the solvent (Table 1).

Transmission electron microscopy (TEM) studies were conducted on carbon coated copper grids (Ted Pella, Inc. 01822-F, support films, ultrathin carbon type-A, 400 mesh copper grid)

Table 1 Reference fluorophores (RF) used to determine quantum yield (QY) values

\begin{tabular}{llllll}
\hline RF & Ex. $\lambda(\mathrm{nm})$ & Solvent & $n^{a}$ & QY $(\%)$ & Ref. \\
\hline QS & 350 & $0.1 \mathrm{M} \mathrm{H}_{2} \mathrm{SO}_{4}$ & 1.343 & 58 & 34 \\
FL & 470 & $0.1 \mathrm{M} \mathrm{NaOH}$ & 1.336 & 91 & 35 \\
RB & 514 & Water & 1.334 & 31 & 36
\end{tabular}

${ }^{a}$ Refractive index. using a FEI Tecnai (F30 G2, Twin) microscope operated at a $300 \mathrm{keV}$ accelerating voltage. Fourier-transform infrared spectra were acquired on a Thermo-Nicolet Nexus 670 ESP FT-IR spectrophotometer using potassium bromide pellets. Circular dichroism spectra were collected on a Jasco J-710 spectropolarimeter and an average of 10 scans were used to produce the spectra. The cyt $c / \mathrm{C}$-dot interactions presented in Fig. 1A were generated using protein modeling software (ePMV) $)^{22-24}$ and an open-source 3D creation suite $\left(\right.$ Blender $\left.^{\mathrm{TM}}\right)$. The charge distribution within cyt $c$ (Fig. 1B) was calculated and modeled using Adaptive PoissonBoltzmann Solver (APBS). ${ }^{23,37}$

\section{Carbon nanodot synthesis}

The carbon nanodots were generated through a thermal pyrolysis approach. In all instances, citric acid (CA) was used as the primary carbon source while various nitrogen- and/or sulfurcontaining compounds (e.g., urea, thiourea, and mercaptosuccinic acid) were employed as the dopant/passivating agent. To generate 'undoped' carbon nanodots CA (42 g) was carbonized alone. For the 'doped' samples, CA was combined with the dopant compound in a 1:3 molar ratio except in the case of mercaptosuccinic acid in which a 1:1 ratio was employed. More specifically, $21 \mathrm{~g}$ of CA was combined with $18 \mathrm{~g}$ of urea, $25 \mathrm{~g}$ of thiourea, or $15 \mathrm{~g}$ of mercaptosuccinic acid to generate the resultant carbon dot samples $\mathrm{CA}-\mathrm{U}, \mathrm{CA}-\mathrm{T}$, and $\mathrm{CA}-\mathrm{M}$, respectively. The precursor combinations described were dissolved in $500 \mathrm{~g}$ of deionized water and were thermally treated at $200{ }^{\circ} \mathrm{C}$ for $24 \mathrm{~h}$ in a round-bottom flask immersed within an improvised sand bath consisting of an anodized aluminum round cake pan (Fat Daddio's) filled with copper-coated airgun BBs (0.177 caliber; Crosman Copperhead). Temperature control was achieved to within $2{ }^{\circ} \mathrm{C}$ using a digital stirring hotplate (Super-Nuova ${ }^{\mathrm{TM}}$; Thermo Scientific $^{\mathrm{TM}}$ ) connected to a J-type thermocouple immersed in the BBs. Stirring was provided at $200 \mathrm{rpm}$ using a PTFE-coated magnetic stir bar. A water addition of $200 \mathrm{~g}$ was made at the $12 \mathrm{~h}$ mark to prevent the samples from scorching. After naturally cooling to room temperature, the resultant thermally-treated solutions were centrifuged at $5000 \mathrm{rpm}$ for $30 \mathrm{~min}$ to remove large particles. The samples were further purified via room temperature dialysis for $48 \mathrm{~h}$ against $1.5 \mathrm{~L}$ of water using $1 \mathrm{kDa}$ MWCO cellulose tubing. The dialysate was replenished after $24 \mathrm{~h}$ and the samples were further dialyzed for an additional $24 \mathrm{~h}$. Finally, the retentates were lyophilized and stored in a lab drawer (protected from light) until needed.

\section{Buffer preparation}

Phosphate buffer (PB) was prepared at a concentration of $10 \mathrm{mM}$ and $\mathrm{pH}$ of 7.37 by dissolving $0.406 \mathrm{~g}$ of sodium phosphate monobasic and $0.9957 \mathrm{~g}$ of sodium phosphate dibasic in $1.0 \mathrm{~L}$ of water (ionic strength, $\mu \approx 0.02 \mathrm{M}$ ). Phosphate buffered saline (PBS) was prepared by dissolving one PBS tablet in $200 \mathrm{~mL}$ of water, resulting in a $\mathrm{pH} 7.4,10 \mathrm{mM}$ phosphate buffer containing $2.7 \mathrm{mM}$ potassium chloride and $137 \mathrm{mM}$ sodium chloride $(\mu \approx 0.16 \mathrm{M})$. These solutions were then used to dissolve lyophilized cyt $c$ and dry ABTS to prepare appropriately concentrated stocks. 


\section{Peroxide-like activity measurements}

Peroxidase-like activities of CA, CA-T, CA-U, and CA-M derived C-dots were tested by monitoring the absorbance of ABTS at $418 \mathrm{~nm}$. In a typical catalytic experiment, the cyt $c$ concentration was $515 \mathrm{nM}$, ABTS concentration was $0.72 \mathrm{mM}, \mathrm{C}-\mathrm{dot}$ concentration was $25.5 \mu \mathrm{g} \mathrm{mL} \mathrm{m}^{-1}$, and peroxide concentration was $14.1 \mathrm{mM}$. To accomplish the study, $20 \mu \mathrm{L}$ of a $1 \mathrm{mg} \mathrm{mL}$ $(80.75 \mu \mathrm{M})$ cyt $c$ stock, $100 \mu \mathrm{L}$ of a $12.35 \mathrm{mg} \mathrm{mL}^{-1}(22.5 \mathrm{mM})$ ABTS stock, and $8 \mu \mathrm{L}$ of a $10 \mathrm{mg} \mathrm{mL}{ }^{-1} \mathrm{C}$-dot solution were added to $3 \mathrm{~mL}$ of water in a quartz cuvette. The mixture was inverted 3 times and allowed to incubate for $30 \mathrm{~min}$ prior to the addition of $5 \mu \mathrm{L}$ of cold $30 \mathrm{wt} \% \mathrm{H}_{2} \mathrm{O}_{2}$. Immediately following the $\mathrm{H}_{2} \mathrm{O}_{2}$ addition, UV-Vis absorbance spectra were collected for a duration of at least $5 \mathrm{~min}$. For the CA C-dot loading optimization, the above approach was also employed, varying the final concentration of $\mathrm{C}$-dots within the cuvette from 0.10 to $50 \mu \mathrm{g} \mathrm{mL}{ }^{-1}$. All other concentrations and volumes were maintained. For the iron control experiments, cyt $c$ was replaced by $\mathrm{Fe}^{2+}$ or $\mathrm{Fe}^{3+}$ at a concentration of $515 \mathrm{nM}$ (identical to the [cyt $c$ ] used previously). All other concentrations and volumes remained

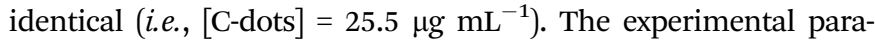
meters for the molecular analog control studies were identical to those used for the initial C-dot screening tests except that, in place of C-dots, model molecules at a final concentration of $2.5 \mu \mathrm{g} \mathrm{mL}$ were used. Finally, the same conditions used for C-dot screening were used in different solvent systems (water, PB, or PBS) to elucidate the interaction between the CA derived C-dots and cyt $c$.

\section{Michaelis-Menten kinetics}

To characterize the peroxidase activity, kinetic measurements were conducted in the presence of CA-derived C-dots for varying ABTS concentrations that ranged from $0.037 \mathrm{mM}$ to $0.718 \mathrm{mM}$. The C-dot, peroxide, and cyt $c$ concentrations were held identical to the experiments described above. The initial velocities were determined by linear regression over the first minute of data collection and were plotted as a function of ABTS concentration. Using these values, a double-reciprocal Lineweaver-Burk plot was generated to attain $V_{\max }, K_{\mathrm{M}}, k_{\text {cat }}$, and catalytic efficiency $\left(k_{\text {cat }} / K_{\mathrm{M}}\right)$ values. To calculate $V_{\max }$ and $K_{\mathrm{m}}$, the equation for the linear fit of the Lineweaver-Burk plot was used. $V_{\max }$ was calculated (eqn (1)) by taking the reciprocal of the $y$-intercept $(b)$, and $K_{\mathrm{M}}$ was calculated (eqn (2)) by multiplying $V_{\max }$ by the slope of the line. $k_{\text {cat }}$ was then calculated by dividing $V_{\max }$ by the protein concentration in the cuvette (eqn (3)). Catalytic efficiency was calculated by dividing $k_{\text {cat }}$ by $K_{\mathrm{M}}($ eqn (4)).

$$
\begin{gathered}
V_{\max }=\frac{1}{b} \\
K_{\mathrm{M}}=V_{\max } \times \text { (slope of line) } \\
k_{\mathrm{cat}}=\frac{V_{\max }}{[E]} \\
\text { Catalytic efficiency }=\frac{k_{\mathrm{cat}}}{K_{\mathrm{M}}}
\end{gathered}
$$

\section{Circular dichroism}

Circular dichroism (CD) measurements were carried out to probe the effects that the C-dots have on the cyt $c$ secondary structure. For the Soret region, a $1 \mathrm{~cm}$ quartz cuvette was used for data collection while for the far-UV region a $1 \mathrm{~mm}$ path length quartz cuvette was used. The concentrations of cyt $c$ and C-dots were $4.17 \mu \mathrm{M}$ and $207 \mu \mathrm{g} \mathrm{mL} \mathrm{m}^{-1}$, respectively. The mixture of cyt $c$ and C-dots was allowed to incubate for $30 \mathrm{~min}$ prior to data collection. Spectra were collected in the Soret region from 300-500 while the UV was collected from 185-250 $\mathrm{nm}$. The helical content was calculated according to the concentration-independent method of Raussens et $a .^{31}$

\section{$695 \mathrm{~nm}$ absorbance band of cyt $c$}

Three separate samples were prepared (CA-derived C-dots, cyt $c$, and cyt $c$ plus CA-derived C-dots) within $3 \mathrm{~mL}$ PMMA cuvettes. The freshly-prepared CA-derived C-dot and cyt $c$ stock concentrations were $10 \mathrm{mg} \mathrm{mL} \mathrm{mL}^{-1}$ and $50 \mu \mathrm{M}$, respectively. A C-dot concentration of $853 \mu \mathrm{g} \mathrm{mL}{ }^{-1}$ was used and the cyt $c$ concentration was $32 \mu \mathrm{M}$. Specifically, $256 \mu \mathrm{L}$ of $10 \mathrm{mg} \mathrm{mL}^{-1} \mathrm{C}$-dots and $1.92 \mathrm{~mL}$ of $50 \mu \mathrm{M}$ cyt $c$ stock solutions were added to two separate cuvettes, followed by dilution to a $3 \mathrm{~mL}$ total volume using deionized water. For the mixed sample, these volumes remained the same and the total volume was again adjusted to $3 \mathrm{~mL}$ using deionized water.

\section{References}

1 W. Shang, J. H. Nuffer, V. A. Muñiz-Papandrea, W. Colón, R. W. Siegel and J. S. Dordick, Small, 2009, 5, 470-476.

2 X. Yang, C. Zhao, E. Ju, J. Ren and X. Qu, Chem. Commun., 2013, 49, 8611-8613.

3 H. Li, S. Guo, C. Li, H. Huang, Y. Liu and Z. Kang, ACS Appl. Mater. Interfaces, 2015, 7, 10004-10012.

4 N. W. S. Kam and H. Dai, J. Am. Chem. Soc., 2005, 127, 6021-6026.

5 S. Radic, P. Nedumpully-Govindan, R. Chen, E. Salonen, J. M. Brown, P. C. Ke and F. Ding, Nanoscale, 2014, 6, 8340-8349.

6 L. Jin, K. Yang, K. Yao, S. Zhang, H. Tao, S.-T. Lee, Z. Liu and R. Peng, ACS Nano, 2012, 6, 4864-4875.

7 T. Kar, S. K. Mandal and P. K. Das, Chem. Commun., 2012, 48, 8389-8391.

8 M. De, S. S. Chou and V. P. Dravid, J. Am. Chem. Soc., 2011, 133, 17524-17527.

9 S. S. Chou, M. De, J. Luo, V. M. Rotello, J. Huang and V. P. Dravid, J. Am. Chem. Soc., 2012, 134, 16725-16733.

10 S. N. Baker and G. A. Baker, Angew. Chem., Int. Ed., 2010, 49, 6726-6744.

11 B. Zheng, T. Liu, M. C. Paau, M. Wang, Y. Liu, L. Liu, C. Wu, J. Du, D. Xiao and M. M. F. Choi, $R S C A d v ., 2015,5$, 11667-11675.

12 J. B. Essner, C. H. Laber, S. Ravula, L. Polo-Parada and G. A. Baker, Green Chem., 2016, 18, 243-250. 
13 S. Huang, H. Qiu, S. Lu, F. Zhu and Q. Xiao, J. Hazard. Mater., 2015, 285, 18-26.

14 T. Han, T. Yan, Y. Li, W. Cao, X. Pang, Q. Huang and Q. Wei, Carbon, 2015, 91, 144-152.

15 S. Li, Z. Peng and R. M. Leblanc, Anal. Chem., 2015, 87, 6455-6459.

16 S. Li, L. Wang, C. C. Chusuei, V. M. Suarez, P. L. Blackwelder, M. Micic, J. Orbulescu and R. M. Leblanc, Chem. Mater., 2015, 27, 1764-1771.

17 S. Mondal, R. Thirupathi and H. S. Atreya, RSC Adv., 2015, 5, 4489-4492.

18 H. Li, W. Kong, J. Liu, M. Yang, H. Huang, Y. Liu and Z. Kang, J. Mater. Chem. B, 2014, 2, 5652-5658.

19 J. B. Essner, C. H. Laber and G. A. Baker, J. Mater. Chem. A, 2015, 3, 16354-16360.

20 M. J. Krysmann, A. Kelarakis, P. Dallas and E. P. Giannelis, J. Am. Chem. Soc., 2011, 134, 747-750.

21 D. Qu, M. Zheng, P. Du, Y. Zhou, L. Zhang, D. Li, H. Tan, Z. Zhao, Z. Xie and Z. Sun, Nanoscale, 2013, 5, 12272-12277.

22 G. T. Johnson, L. Autin, D. S. Goodsell, M. F. Sanner and A. J. Olson, Structure, 2011, 19, 293-303.

23 M. F. Sanner, A. J. Olson and J.-C. Spehner, Biopolymers, 1996, 38, 305-320.

24 D. Frishman and P. Argos, Proteins, 1995, 23, 566-579. 25 P. A. Mabrouk, Bioconjugate Chem., 1994, 5, 236-241.
26 S. Debnath, D. Das and P. K. Das, Biochem. Biophys. Res. Commun., 2007, 356, 163-168.

27 A. Ranieri, F. Bernini, C. A. Bortolotti, A. Bonifacio, V. Sergo and E. Castellini, Langmuir, 2011, 27, 10683-10690.

28 R. E. M. Diederix, M. Ubbink and G. W. Canters, Eur. J. Biochem., 2001, 268, 4207-4216.

29 R. E. M. Diederix, M. Ubbink and G. W. Canters, ChemBioChem, 2002, 3, 110-112.

30 N. J. Greenfield, Nat. Protoc., 2006, 1, 2876-2890.

31 V. Raussens, J.-M. Ruysschaert and E. Goormaghtigh, Anal. Biochem., 2003, 319, 114-121.

32 G. A. Baker and W. T. Heller, Chem. Eng. J., 2009, 147, 6-12.

33 C. Madden, M. D. Vaughn, I. Díez-Pérez, K. A. Brown, P. W. King, D. Gust, A. L. Moore and T. A. Moore, J. Am. Chem. Soc., 2012, 134, 1577-1582.

34 J. R. Lakowicz, Principles of Fluorescence Spectroscopy, Springer, 2007.

35 L. Porrès, A. Holland, L.-O. Pålsson, A. Monkman, C. Kemp and A. Beeby, J. Fluoresc., 2006, 16, 267-273.

36 D. Magde, G. E. Rojas and P. G. Seybold, Photochem. Photobiol., 1999, 70, 737-744.

37 N. A. Baker, D. Sept, S. Joseph, M. J. Holst and J. A. McCammon, Proc. Natl. Acad. Sci. U. S. A., 2001, 98, 10037-10041. 